\title{
The Photon Detector and the Variable-Pressure Scanning Electron Microscope: An Alternative Method for Cathodoluminescence Imaging of Geologic Materials
}

\author{
T.J. Williams, * J.F. Bailey, $* *$ \\ * Department of Geological Sciences, University of Idaho, Moscow, ID 83844 \\ ** Electron Microscopy Center, University Research Office, University of Idaho, Moscow, Id 83844
}

Minerals are rarely homogeneous and often display multiple generations of crystal growth and complex chemical zonation that are difficult to observe with standard microscopic techniques. The advent of new in situ methods of chemical and isotopic analysis [U-Pb dating by SHRIMP and laserablation ICP-MS trace element analysis] has led to the routine application of cathodoluminescence imaging (CL) of minerals such as zircon $\left[\mathrm{ZrSiO}_{4}\right]$, quartz $\left[\mathrm{SiO}_{2}\right]$, and calcite $\left[\mathrm{CaCO}_{3}\right]$ to map complex internal structures. A common CL technique in the geosciences is the "hot cathode" method utilizing a SEM or EPMA equipped with a dedicated photo-multiplier tube (PMT) CL detector.

Using a Zeiss Supra $35{ }^{\mathrm{TM}}$ Variable-Pressure Field Emission SEM [VP FESEM], we observed that a properly-equipped variable-pressure SEM (VP SEM) may offer a workable substitute for a dedicated CL detection system. The VP SEM is designed to generate secondary electron images at relatively high pressures (10-2500 $\mathrm{Pa})$ versus a standard SEM which requires a high operating vacuum $\left(10^{-3} \mathrm{~Pa}\right.$ or greater). VP SEMs utilize a variety of detector technologies to generate a high pressure secondary electron image [1]. The Supra $35^{\mathrm{tm}}$ VP FESEM at the University of Idaho is equipped with a variable pressure secondary electron detector (VP SE) that collects photons generated by cascade collisions between secondary electrons and gas molecules within the sample chamber. When a luminescent sample is imaged under routine high voltage conditions the VP SE photon detector functions as a monochromatic CL detector.

For comparison purposes we collected a CL images of zircons from northern Idaho [Fig. 1] utilizing our AMRAY 1830 SEM [tungsten filament] equipped with a dedicated PMT-based CL detector [Gatan miniCL ${ }^{\mathrm{TM}}$ ], and the Supra $35^{\mathrm{tm}}$ VP FESEM utilizing the VP SE photon detector [Fig. 2]. Digital images were collected under similar beam $(15 \mathrm{kV})$, vacuum $\left(10^{-3} \mathrm{~Pa}\right)$ and frame-buffering conditions.

The quality and usefulness of monochromatic CL images are subjective. The objective is to produce images with sufficient information for use with other micro beam techniques [i.e. laser-ablation ICPMS]. Acceptability depends on the judgment and skill of the operator. In our comparative study, gray-scale sensitivity was lower in the VP SE CL images on the Supra $35^{\mathrm{tm}}$ as compared to CL images collected on the AMRAY with the miniCL ${ }^{\mathrm{tm}}$ detector but otherwise were of comparable resolution and quality [Fig. 1]. Although designed for the collection of secondary electron images, the VP SE detector functions well for the collection of CL images. The growing popularity of the VP SEM makes them a potentially untapped resource for CL imaging. The caveat here is that not all VP SEMs use a photon-based detector for VP SE imaging. For reasons of initial instrument cost and ease of use, a dedicated CL detector system on a SEM or EPMA is preferred as a method for routine CL imaging. However, when this tool is unavailable, a VP SEM with a VP SE photon detector offers a practical alternative. 


\section{References}

[1] J.I. Goldstein et al., Scanning Electron Microscopy and X-ray Microanalysis, Plenum, New York, 2003.

[2] The Murdock Charitable Trust provided support for the Field-Emission VP SEM Laboratory. The assistance of Dr. William McClelland is gratefully acknowledged.
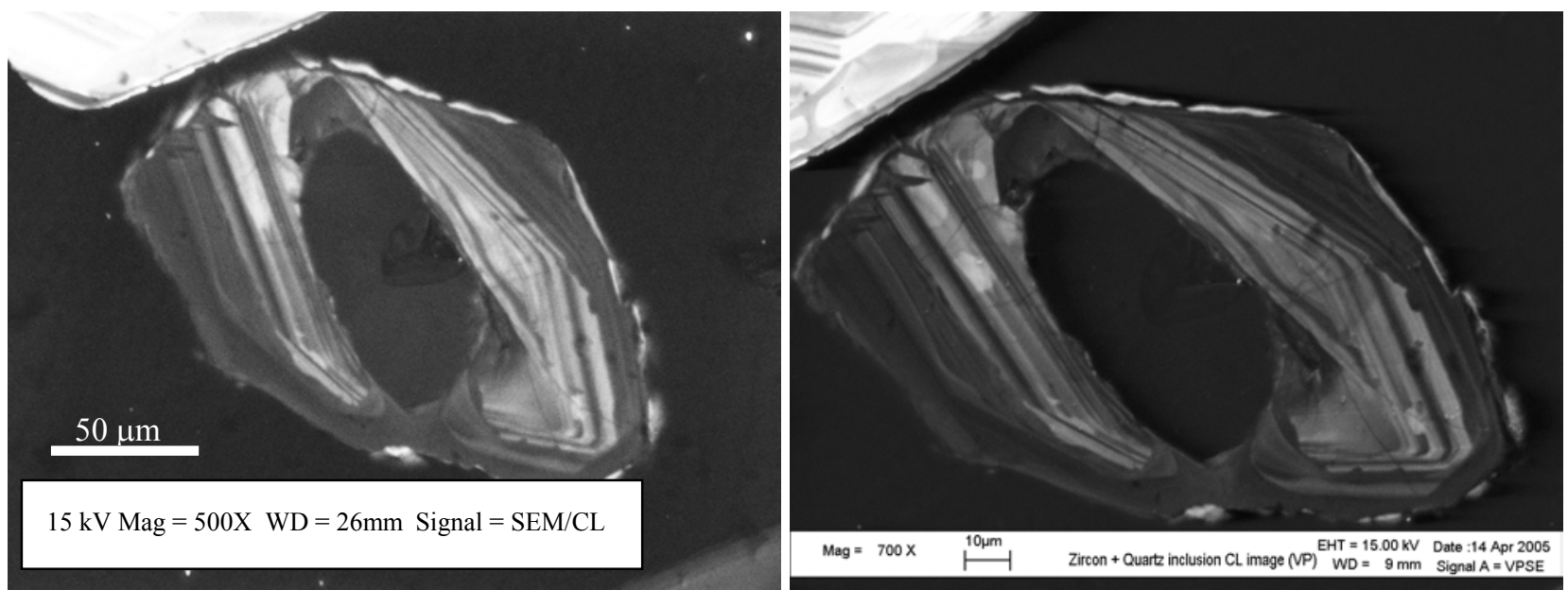

FIG. 1. SEM/CL images of a single Zircon crystal [w/quartz core] collected with Gatan miniCL ${ }^{\mathrm{tm}}$ PMT CL detector on the AMRAY 1830 [right], and the VP SE photon detector on Supra $35^{\mathrm{tm}}$ VP FESEM [left].
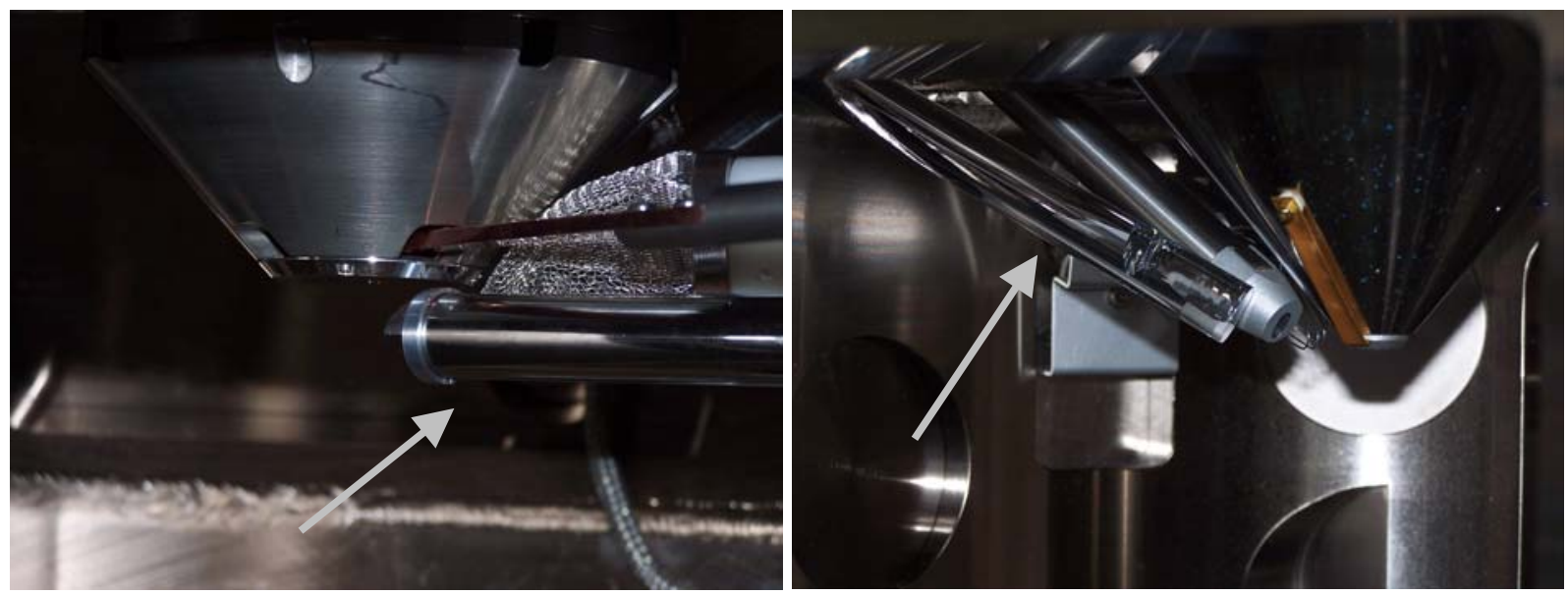

FIG. 2. [right] The Gatan miniCL ${ }^{\mathrm{tm}}$ PMT CL detector [see arrow] on the AMRAY 1830, and the VP SE photon detector [left] on the Zeiss Supra $35^{\mathrm{tm}}$ VP FESEM [see arrow]. 